\title{
Downregulation of microRNA-138 enhances the proliferation, migration and invasion of cholangiocarcinoma cells through the upregulation of RhoC/p-ERK/MMP-2/MMP-9
}

\author{
QI WANG, HUIHUAN TANG, SHANSHAN YIN and CHAO DONG \\ Xiangya Hospital, Central South University, Changsha, Hunan 410008, P.R. China
}

Received November 16, 2012; Accepted January 25, 2013

DOI: $10.3892 /$ or.2013.2304

\begin{abstract}
RNAs (miRs) play an important role in tumor initiation and progression in many types of cancer, including cholangiocarcinoma (CC). miR-138 dysregulation is frequently observed in a variety of tumors. In the present study, miR-138 was found to be downregulated in CC tissues by quantitative real-time RT-PCR. Furthermore, its potential target molecule, Ras homolog gene family, member $\mathrm{C}$ (RhoC) protein, was found to be highly expressed in $\mathrm{CC}$ tissues examined by western blot analysis. Luciferase reporter assay further demonstrated that miR-138 directly targeted RhoC. We found that the introduction of miR-138 mimics to RBE and QBC939 CC cells could reduced RhoC mRNA and protein expression, and suppressed the proliferation, G1/S transition, migration and invasion of CC cells. However, transfection with a miR-138 inhibitor induced an inverse effect in CC cells. The expression of phosphorylated extracellular signal-regulated kinase (p-ERK), matrix metalloproteinase (MMP)-2 and MMP-9 decreased following transfection with miR-138, and increased following transfection with miR-138 inhibitor in CC cells. In conclusion, RhoC upregulation induced by miR-138 downregulation promotes the malignant progression of CC cells and the underlying mechanisms of this effect involve the increase in the expression of p-ERK/MMP-2/MMP-9. Consequently, miR-138/RhoC is a potential target for the clinical diagnosis and treatment of $\mathrm{CC}$.
\end{abstract}

\section{Introduction}

Cholangiocarcinoma (CC), primary bile duct cancer, divided into intrahepatic and extrahepatic tumors according to its location, is a rare and highly malignant tumor (1). Risk factors include hepatitis $\mathrm{B}$ and $\mathrm{C}$, cirrhosis and alcohol consumption. The incidence of CC is increasing globally (2-4). Recently, an increasing

Correspondence to: Professor Huihuan Tang, Xiangya Hospital, Central South University, 87 Xiangya Road, Changsha, Hunan 410008, P.R. China

E-mail: 5309433@qq.com

Key words: cholangiocarcinoma, microRNA-138, RhoC, proliferation, migration, invasion number of studies have focused on a new class of small noncoding regulatory RNA molecules, termed microRNAs (miRs, miRNAs). miRs are highly conserved among species and regulate the expression of specific target genes by binding to the 3'-untranslated regions (3'-UTRs) of messenger RNA (mRNA). miR dysregulation has been implicated in tumor initiation and progression in many types of cancer, including CC (5-7). Studies have shown that miR-421 (8), miR-26a (9), miR-494 (10), miR-25 (11), miR-200c (12), miR-21 (13-15), miR-214 (16), miR-373 (17-19), miR-494 (20) and miR-370 (21) are involved in the regulation of cell growth, cell cycle distribution, apoptosis and metastasis in CC.

The downregulation of miR-138 is frequently observed in a variety of tumors (22-26). miR-138 is able to target a variety of genes, such as $\mathrm{G}$ protein $\alpha$ inhibiting activity polypeptide 2 in tongue squamous cell carcinoma (27), cyclin D3 in hepatocellular carcinoma (28), cyclin D1 in nasopharyngeal carcinoma (29), Rho-associated, coiled-coil-containing protein kinase 2 (ROCK2) and Ras homolog gene family, member C (RhoC) in tongue squamous cell carcinoma (23), as well as others. It is well known that Ras homolog (Rho) family GTPases, including RhoC play a pivotal role in tumor malignant transformation and metastasis $(30,31)$. Knockdown of the RhoC gene suppresses the proliferative and invasive capacity of human QBC939 CC cells (32). However, the precise regulatory effects of miR-138 on a gene [for example, Rho guanine nucleotide exchange factor (GEF)3 ARHGEF3] may vary depending on the cell type (23). Thus far, the expression pattern of miR-138 and RhoC in CC tissues, and whether miR-138 is involved in the progression of CC remain unknown.

In the present study, the expression levels, the functional roles and the correlation between miR-138 and RhoC in CC were investigated. It was confirmed that the miR-138/RhoCmediated progression of $\mathrm{CC}$ involves the upregulation of the expression of extracellular signal-regulated kinase (ERK)/ matrix metalloproteinase (MMP)-2/MMP-9.

\section{Materials and methods}

Cell culture and transfection. RBE and CQB939 CC cell lines were purchased from the China Center for Type Culture Collection (CCTCC, Wuhan, China) and were maintained in RPMI-1640 (HyClone) containing 10\% fetal bovine serum 
(Hangzhou Sijiqing) in a humidified atmosphere of $5 \% \mathrm{CO}_{2}$ at $37^{\circ} \mathrm{C}$. For functional analysis, RBE and CQB939 cells were transfected with scrambled control miRNA (negative control, NC), miR-138 mimics, or miR-138 inhibitor (Ambion) using Lipofectamine 2000 (Invitrogen) according to the manufacturer's recommendations.

Tumor tissue sample preparation. A total of 9 patients diagnosed with stage Ib, IIa or IIb CC were recruited in our study. The experimental protocols were approved by the Ethics Committee of the Xiangya Hospital, Central South University, Changsha, China. RNA or protein samples prepared from the tumor tissues and their matched adjacent non-tumor tissues were then subjected to quantitative real-time RT-PCR and western blot analysis.

Real-time RT-PCR. Total RNA was extracted from the cells or tissue samples with TRIzol reagent (Invitrogen) following the manufacturer's instructions. The relative expression level of miR-138 was determined by quantitative real-time RT-PCR using the mirVana ${ }^{\mathrm{TM}}$ qRT-PCR microRNA Detection kit (Ambion) following the manufacturer's instructions. Specific primer sets for miR-138 and U6 (used as an internal reference) were obtained from Ambion. The expression of RohC mRNA was detected by real-time RT-PCR using the standard SYBRGreen RT-PCR Kit (Takara, Otsu, Japan) following the manufacturer's instructions. The specific primer pairs were as follows: RhoC (161 bp) sense, 5'-AAGGACCTGAGGCAA GACGAGC-3' and antisense, 5'-TCAAACACCTCCCGC ACTCCC-3'; $\beta$-actin (202 bp; used as an internal control) sense, 5'-AGGGGCCGGACTCGTCATACT-3' and antisense, 5'-GGCGGCACCACCATGTACCCT-3'. The relative expression of RhoC mRNA or miR-138 was quantified using GraphPad Prism 4.0 software (GraphPad Software, San Diego, CA, USA) and the $2^{-\Delta \Delta \mathrm{Ct}}$ method (33).

Dual luciferase reporter assay. The 3'-UTR of RhoC (position 1210 to 1271, NM_175744) containing the miRNA-138 binding sites and its corresponding mutated sequence were cloned into the psi-CHECK2 luciferase reporter vector (Promega) downstream of Renilla luciferase, termed 3'-UTR RhoC and 3'-UTR Mut RhoC, respectively. Using Lipofectamine 2000 (Invitrogen), human embryonic kidney (HEK)293T cells were co-transfected with the reporter constructs and miR-138 mimics, negative control or miR-138 inhibitor (Ambion). Luciferase activity was determined after $48 \mathrm{~h}$ using the Dual-Glo substrate system (Promega) and a Beckman Coulter LD400 luminometer. Data are presented as the ratio of experimental (Renilla) luciferase to control (firefly) luciferase.

Cell viability assay. Cells in exponential growth were plated at a final concentration of $2 \times 10^{3}$ cells/well in 96-well plates. Cell viability was assessed by MTT assay after 24, 48, 72 and $96 \mathrm{~h}$ of seeding. The optical density at $570 \mathrm{~nm}$ (OD570) of each well was measured with an ELISA reader (ELx-800 type; BioTek).

Cell cycle analysis by flow cytometry (FCM). The cells were digested and collected at $48 \mathrm{~h}$ post-transfection and washed with PBS twice. The cells were resuspended in PBS and then fixed in $70 \%$ ethanol at $4^{\circ} \mathrm{C}$ for $18 \mathrm{~h}$. The cells were washed with PBS and resuspended in staining solution $(50 \mu \mathrm{g} / \mathrm{ml}$ of propidium iodide, $1 \mathrm{mg} / \mathrm{ml}$ of RNase A, $0.1 \%$ Triton X-100 in PBS). The stained cells $\left(1 \times 10^{5}\right)$ were then analyzed with a flow cytometer (Beckman Coulter).

Cell migration assay. Cell migration was measured using a wound healing assay as described by Saadoun et al (34). In brief, cells transfected with miRNAs or inhibitor as indicated, were cultured to confluence. Wounds of $\sim 1 \mathrm{~mm}$ width were created with a plastic scriber, and the cells were washed and incubated in serum-free medium. After wounding for $24 \mathrm{~h}$, cells were incubated in medium including $10 \%$ fetal bovine serum. Cultures at 0,24 and $48 \mathrm{~h}$ were fixed and observed under a microscope.

Cell invasion assay. The cell invasion assay was performed using a Cell Invasion Assay kit (Chemicon International, Temecula, CA, USA) according to the manufacturer's instruction as previously described by Aspenstrom et al (35). Briefly, the cells were placed in the upper compartment of the chambers, and RPMI-1640 containing $10 \%$ fetal bovine serum was added to the lower chambers. After $24 \mathrm{~h}$ of incubation at $37^{\circ} \mathrm{C}$, cells on the upper face of the membrane were scraped using a cotton swab and cells on the lower face were fixed, stained and observed under a microscope. The dye on the membrane was then dissolved with $10 \%$ acetic acid, dispensed into 96-well plates (150 $\mu \mathrm{l} /$ well), and the OD570 of each well was measured with an ELISA reader (ELx-800 type; BioTek).

Western blot analysis. Cells were lysed in cell lysis buffer containing $7 \mathrm{M}$ Urea, $2 \mathrm{M}$ Thiourea, $60 \mathrm{mM}$ DTT, 4\% CHAPS, $2 \%$ pharmalyte $3-10,1.4 \mathrm{mg} / \mathrm{ml}$ PMSF, then centrifuged at $12,000 \mathrm{x} \mathrm{g}$ for $20 \mathrm{~min}$ at $4^{\circ} \mathrm{C}$. The supernatant was collected and denatured. Proteins were separated in 10\% SDS-PAGE and blotted onto polyvinylidene difluoride membranes (PVDF). The PVDF membranes were treated with TBST containing $50 \mathrm{~g} / \mathrm{l} \mathrm{skimmed} \mathrm{milk} \mathrm{at} \mathrm{room} \mathrm{temperature} \mathrm{for} 4 \mathrm{~h}$, followed by incubation with the primary antibodies, anti-RhoC, antiphosphorylated ERK (p-ERK), anti-total-ERK, anti-MMP-2, anti-MMP-9 and anti- $\beta$-actin (Santa Cruz Biotechnology, Santa Cruz, CA, USA), at $37^{\circ} \mathrm{C}$ for $1 \mathrm{~h}$. The membranes were rinsed and incubated for $1 \mathrm{~h}$ with the correspondent peroxidase-conjugated secondary antibodies. Chemiluminescent detection was performed using the ECL kit (Pierce Chemical Co., Rockford, IL, USA). The amount of the protein of interest, expressed as arbitrary densitometric units, was normalized to the densitometric units of $\beta$-actin.

Statistical analysis. Data are expressed as the means \pm SD from at least 3 separate experiments. Statistical analysis was carried out using SPSS 11.0 software. Differences between 2 groups were analyzed by the Student's t-test. A value of $\mathrm{P}<0.05$ was considered to indicate a statistically significant difference.

\section{Results}

Expression of miR-138 and RhoC in CC tissues or cells. Real-time quantitative RT-PCR was performed to detect the expression of miR-138 in CC (stage Ib, IIa or IIb) and adjacent tissues. Compared to the adjacent non-tumor tissues, the 

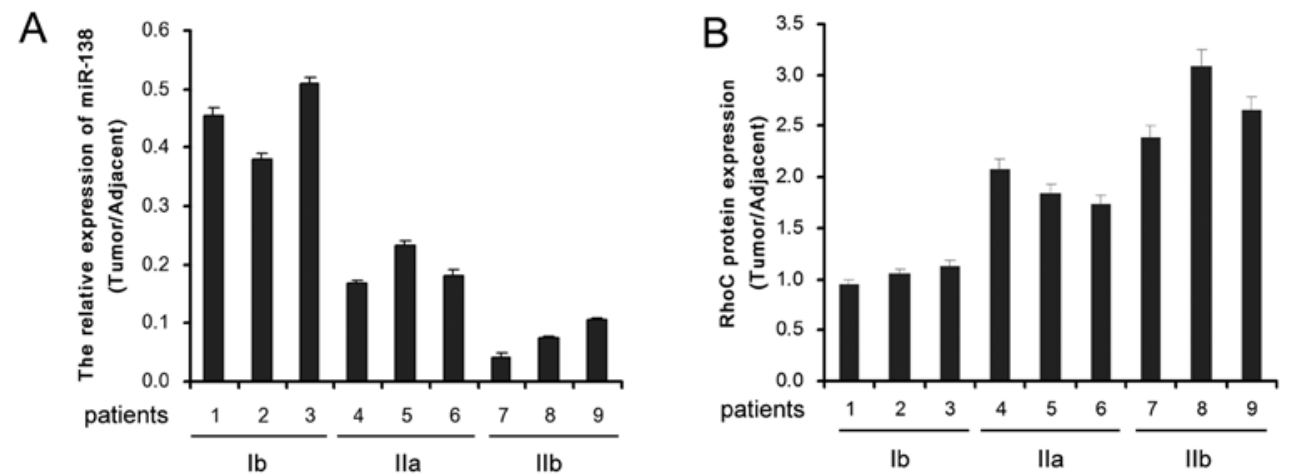

Figure 1. The expression levels of (A) miR-138 and (B) RhoC in cholangiocarcinoma tissues (stage Ib, IIa and IIb), adjacent tissues and normal tissues, identified using real-time RT-PCR or western blot analysis. Data shown are the means \pm SD of a representative experiment performed in triplicate.

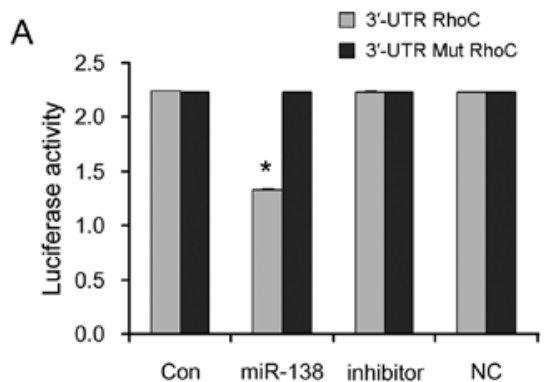

C

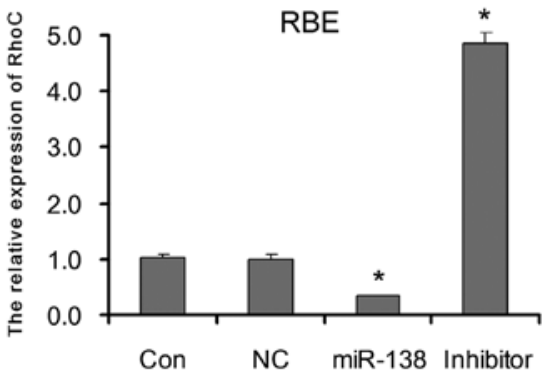

$\mathrm{E}$

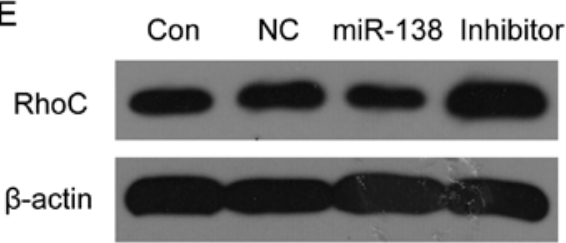

B

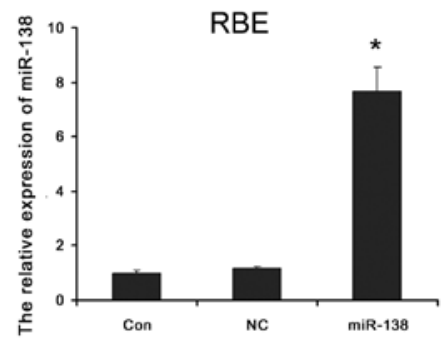

D

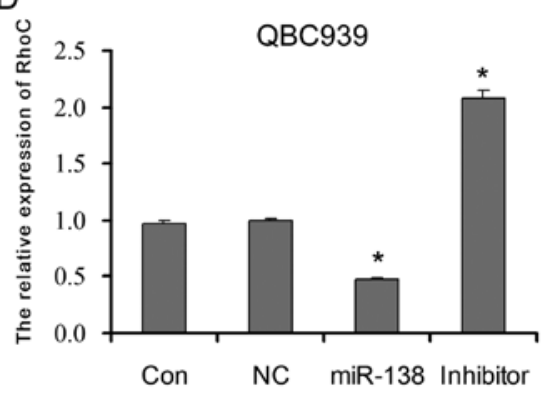

QBC939

$\mathrm{F}$

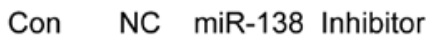

RhoC

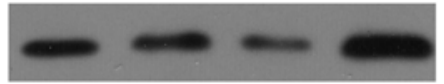

$\beta$-actin

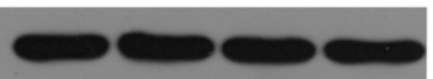

Figure 2. RhoC is a direct target of miR-138. (A) Dual luciferase reporter assays were performed to examine the interaction of miR-138 and its targeting sequence in the RhoC 3'-UTR using constructs containing the targeting sequence (termed 3'-UTR RhoC) and mutated targeting sequence (termed 3'-UTR Mut RhoC) cloned into psi-CHECK2. The relative expression levels of (B) miR-138 in RBE cells and RhoC mRNA in (C) RBE cells and (D) QBC939 cells were detected by real-time RT-PCR. The protein levels of RhoC in (E) RBE and (F) QBC939 CC cells transfected with miR-138 mimics, miR-138 inhibtor (inhibitor) or negative control (NC) of miR-138 were analyzed by western bolt analysis. Data shown are the means \pm SD of a representative experiment performed in triplicate. ${ }^{*} \mathrm{P}<0.05$ vs. control (Con)

expression level of miR-138 in CC tissues was significantly reduced and decreased progressively along with malignant progression (Fig. 1A). Inversely, the expression level of RhoC was significantly enhanced in $\mathrm{CC}$ tissues and increased progressively along with malignant progression (Fig. 1B). Thus, a negative correlation may exist between miR-138 and RhoC in $\mathrm{CC}$ progression. These results indicate that miR-138 upregulation and $\mathrm{RhoC}$ downregulation are involved in the malignant progression of CC.
RhoC is a direct target of miR-138 in CC. Our data from dual luciferase reporter assay showed that the luciferase activity was significantly inhibited only in the HEK293T cells co-transfected with miR-138 mimics and the 3'-UTR RhoC reporter vector. There were no distinct differences in luciferase activity between the other co-transfected cells and the control cells transfected only with 3'-UTR or 3'-UTR mutation RhoC reporter vector (Fig. 2A). These results demonstrated that miR-138 directly targeted RhoC. 
A
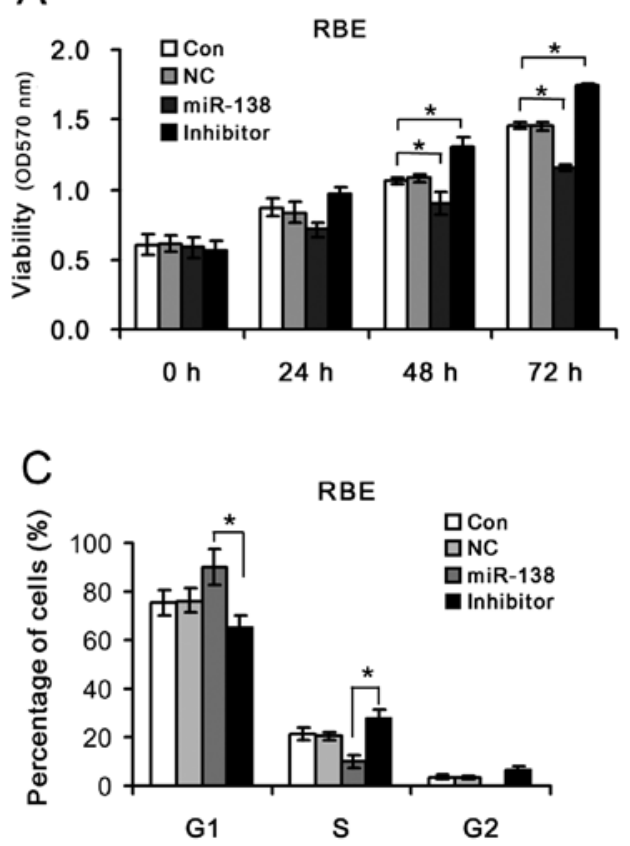

E
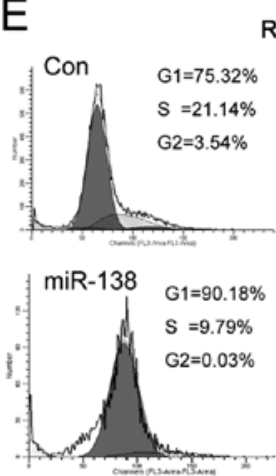

RBE
$\mathrm{B}$
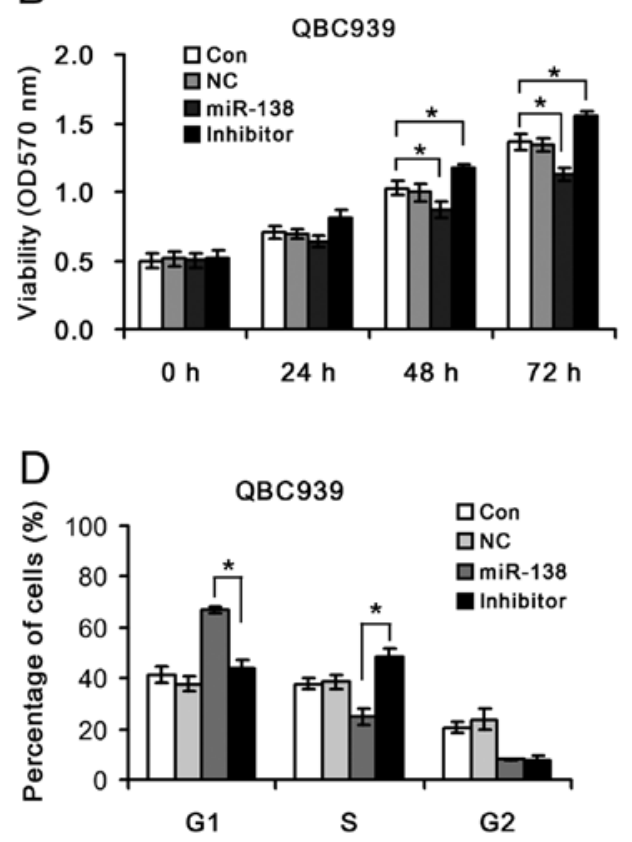

$\mathrm{F}$

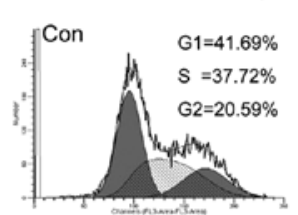

QBC939
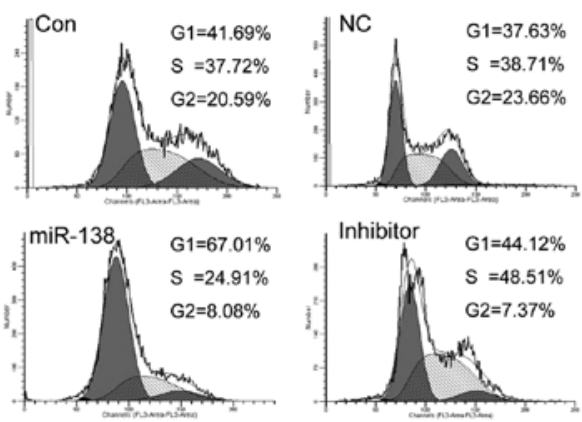

Figure 3. Effects of miR-138/RhoC on cell viability and cell cycle distribution. The cholangiocarcinoma cells were transfected with miR-138 mimics, miR-138 inhibtor (inhibitor) or negative control (NC) of miR-138. The cell viability (A and B) from 0 to $72 \mathrm{~h}$ and cell cycle distribution (C, D, E and F) at $48 \mathrm{~h}$ were measured by MTT assay and FCM, respectively. Representative FCM results (E and F) showing the cell cycle distribution in different transfected CC cells. Data shown are the means $\pm \mathrm{SD}$ of a representative experiment performed in triplicate. ${ }^{*} \mathrm{P}<0.05$.

To confirm the regulatory effect of miR-138 on RhoC in CC cells, the expression levels of RhoC mRNA and protein in the RBE and QBC939 CC cells transfected with miR-138 mimics (which successfully enhanced the miR-138 level in CC cells as shown in Fig. 2B), miR-138 inhibitor or negative control (NC) miR-138 were detected. The results showed that the introduction of miR-138 mimics to RBE and QBC939 CC cells reduced RhoC mRNA (Fig. 2C and D) and protein (Fig. 2D and E) expression; however, miR-138 inhibitor increased RhoC mRNA and protein expression levels. This suggests that miR-138 serves as a suppressor of RhoC by directly targeting RhoC in CC cells.

Effect of miR-138/RhOC on cell viability and cell cycle distribution. To investigate the effects of miR-138/RhoC on cell viability and cell cycle distribution in CC, the RBE and QBC939 CC cells were transfected with miR-138 mimics or miR-138 inhibitor. Cell viability significantly decreased in the RBE and QBC939 CC cells transfected with miR-138 mimics, but markedly increased following transfection with miR-138 inhibitor compared to the control (Fig. 3C). The percentages of G1 phase cells in the miR-138 mimics group were significantly higher than those in the miR-138 inhibitor group. The introduction of miR-138 mimics enhanced the G1/S ratio in the RBE and QBC939 CC cells (9.21 and 2.69, respectively); however, the inhibition of miR-138 reduced the G1/S ratio in the RBE and QBC939 CC cells (2.35 and 0.91, respectively) (Fig. 3D and E). This suggests that the miR-138-mediated RhoC up- or downregulation plays an important role in the regulation of CC cell survival by controlling cell proliferation and $\mathrm{G} 1 / \mathrm{S}$ transition.

Effect of miR-138/RhOC on cell migration and invasion. In order to determine the effects of miR-138/RhoC on CC metastasis, the migration and invasive capability of RBE and QBC939 CC cells transfected with miR-138 mimics or inhibitor were examined. We found that compared to the control, introducing miR-138 mimics to the RBE and QBC939 $\mathrm{CC}$ cells significantly inhibited their migration and invasion. However, migration and invasion were enhanced with the 


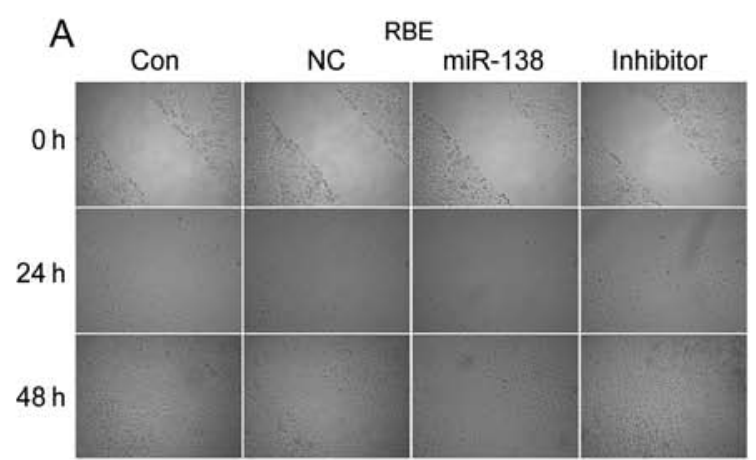

C

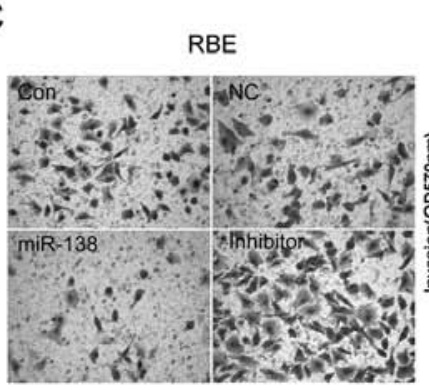

D

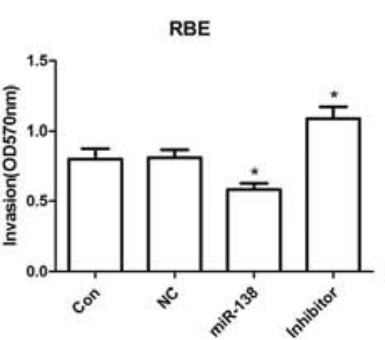

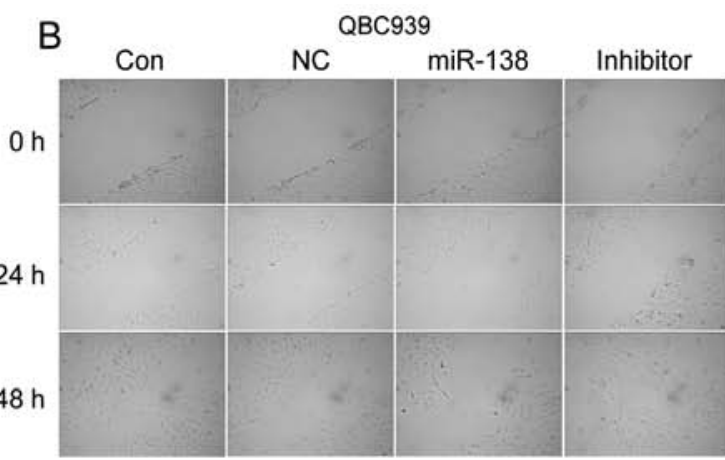

E

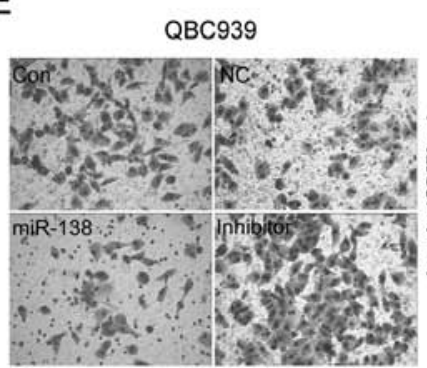

$\mathrm{F}$

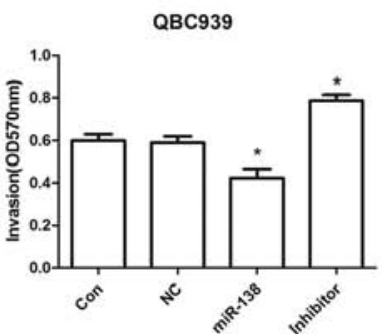

Figure 4. Effects of miR-138/RhoC on CC cell migration and invasion. The migration (A and B) and invasion (C, D, E and F) capabilities were measured by wound healing assay and transwell assay, respectively. Representative wound healing assay (A and B) and transwell assay (C and E) results showing the cell migration and invasion capabilities in different transfected CC cells. Data shown are the means \pm SD of a representative experiment performed in triplicate. ${ }^{*} \mathrm{P}<0.05$ vs. control (Con).

A p-ERK Con NC miR-138 Inhibitor

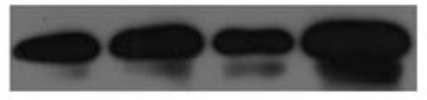

ERK

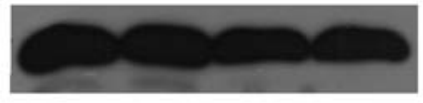

MMP-2

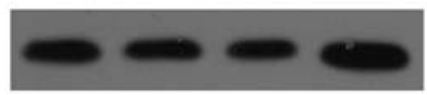

MMP-9

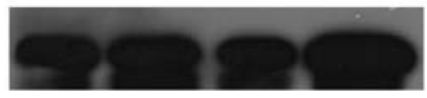

$\beta$-actin
B

p-ERK

ERK
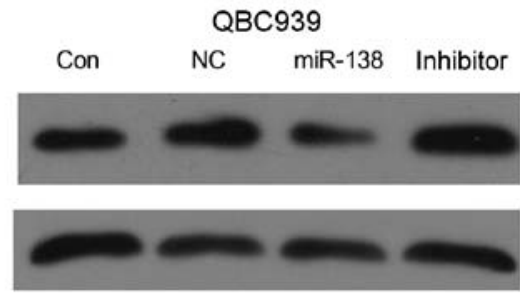

MMP-2

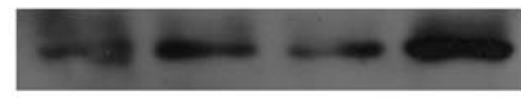

MMP-9

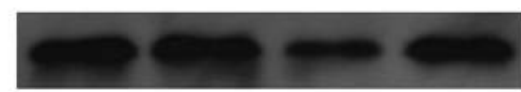

$\beta$-actin

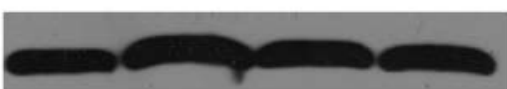

Figure 5. Effects of miR-138/RhoC on ERK, p-ERK, MMP-2 and MMP-9 expression. The protein levels of phosphorylated ERK, total ERK, MMP-2 and MMP-9 in transfected (A) RBE and (B) QBC939 CC cells were analyzed by western bolt analysis. Data represent at least 3 independent experiments with similar results.

inhibition of miR-138 in RBE and QBC939 CC cells (Fig. 4). These results suggest that the upregulation of RhoC caused by the downregulation of miR-138 promotes CC cell migration and invasion. In other words, it contributes to the malignant transformation of CC.

Effect of miR-138/RhoC on ERK, $p$-ERK, MMP-2 and MMP-9 expression. To determine which molecules are involved in the regulation of migration and invasion induced by the dysregulation of miR-138/RhoC in CC, the protein levels of p-ERK, ERK,
MMP-2 and MMP-9 were analyzed by western blot analysis. The results showed that introducing miR-138 mimics to the RBE and QBC939 CC cells decreased p-ERK (without effects on total ERK), MMP-2 and MMP-9 expression. Inversely, the inhibition of miR-138 in RBE and QBC939 CC cells increased p-ERK (without effects on total ERK), MMP-2 and MMP-9 protein expression (Fig. 5). This indicates that RhoC upregulation caused by miR-138 downregulation promotes CC cell migration and invasion. The underlying mechanisms involve the increase in the expression of p-ERK, MMP-2 and MMP-9. 


\section{Discussion}

Tumor metastasis is considered the dominant cause of mortality in cancer patients. Current therapeutic approaches for highly invasive cholangiocarcinoma are not satisfactory. Therefore, efforts to elucidate the molecular mechanisms underlying the metastatic process are of significant clinical importance. Our data from the comparison of tumor tissues from patients with different stages of the disease and adjacent non-tumor tissues showed that the miR-138 expression level gradually decreased with the enhancement of metastatic potential in CC tissues and the corresponding RhoC level increased progressively. Therefore, understanding the functions of miR-138 and RhoC, and their correlation is important for the clinical diagnosis and treatment of CC. We then demonstrated the post-transcriptional regulation of RhoC by miR-138 through the direct binding to its 3'-UTR using dual luciferase reporter assay. The transfection of RBE and QBC939 CC cells with miR-138 mimics resulted in a significant increase in the miR-138 level and a decrease in RhoC mRNA and protein levels. These data suggest that miR-138 downregulation induces RhoC upregulation in CC and this process is likely involved in CC progression.

To confirm the functions of miR-138 and RhoC in CC cells, a series of experiments were performed, demonstrating that the increased miR-138 expression level suppressed the proliferation, G1/S transition, migration and invasion of CC cells, and decreased the expression of RhoC, p-ERK, MMP-2 and MMP-9 in CC cells. However, the upregulation of RhoC by the inhibition of miR-138 in CC cells increased the proliferation, G1/S transition, migration and invasion of the cells, and increased the expression of p-ERK, MMP-2 and MMP-9. These data suggest that RhoC upregulation caused by miR-138 downregulation plays an important role in cell growth regulation, cell cycle transition and metastasis of $\mathrm{CC}$.

A number of studies have revealed that RhoC plays an important role in cell proliferation, cycle distribution and tumor metastasis $(23,32)$. It has been reported that decreasing the RhoC expression level by transfection with antisense RhoC cDNA inhibits proliferation and increases the percentage of CC cells in the G1 phase (32). Our results also indicated that RhoC downregulation promoted by transfection with miR-138 mimics suppressed the proliferation and G1/S transition in CC cells. The inhibition of ERK phosphorylation induced by RhoC downregulation in RBE and QBC939 CC cells transfected with miR-138 mimics suppressed cyclin D1 expression, which plays an important role in the regulation of G1/S transition and cell proliferation $(36,37)$. However, whether this process was partly controlled through miR-138 directly targeting cyclin D1 (29) or cyclin D3 (28), which are 2 of the the target genes of miR-138, requires further confirmation.

Cell migration and invasion are initial steps in tumor cell metastasis. Our data indicated that the inhibition of miR-138 in $\mathrm{CC}$ cells or the increase in the $\mathrm{RhoC}$ expression level due to miR-138 downregulation in CC tumor tissues may result in the enhanced migration and invasion of CC. These effects may be linked to the increased p-ERK, MMP-2 and MMP-9 expression induced by RhoC upregulation in RBE and QBC939 CC cells transfected with miR-138 mimics $(38,39)$. Studies have indicated that RhoC upregulation can promote actin stress fiber and focal adhesion contact formation and may thus facilitate
CC cell migration $(23,40)$. The upregulation of MMP-2 and MMP-9 can cause the CC cells to break through the surrounding tissue barrier via enzymatic degradation of the extracellular matrix (41). Moreover, the activation of ERK possibly enhances MMP-2 and MMP-9 release from CC cells (42). In addition, it has been reported that the upregulation of RhoC and p-ERK expression promotes VEGF expression, resulting in accelerated angiogenesis and distant metastasis (43). These data suggest that $\mathrm{RhoC}$ upregulation caused by miR-138 downregulation promotes CC malignant transformation. The mechanisms underlying these effects involve the activation of ERK and the upregulation of MMP-2 and MMP-9 expression.

In conclusion, $\mathrm{RhoC}$ is highly expressed in $\mathrm{CC}$ tissues and its expression gradually increases with the downregulation of miR-138. RhoC is a direct target of miR-138 in CC. The upregulation of miR-138 suppresses the proliferation, G1/S transition, migration and invasion of RBE and QBC939 CC cells. The potential mechanisms involved include the inhibition of RhoC expression, ERK activation, and the downregulation of MMP-2 and MMP-9 expression. Therefore, it can be concluded that RhoC upregulation induced by miR-138 downregulation plays an important role in the malignant progression of $\mathrm{CC}$, and $\mathrm{miR}-138 / \mathrm{RhoC}$ is a potential target for the clinical diagnosis and treatment of CC.

\section{References}

1. Shimoda M and Kubota K: Multi-disciplinary treatment for cholangiocellular carcinoma. World J Gastroenterol 13: 1500-1504, 2007.

2. Patel T: Cholangiocarcinoma. Nat Clin Pract Gastroenterol Hepatol 3: 33-42, 2006.

3. Reddy SB and Patel T: Current approaches to the diagnosis and treatment of cholangiocarcinoma. Curr Gastroenterol Rep 8: 30-37, 2006.

4. Singh $\mathrm{P}$ and Patel T: Advances in the diagnosis, evaluation and management of cholangiocarcinoma. Curr Opin Gastroenterol 22: 294-299, 2006.

5. Kawahigashi Y, Mishima T, Mizuguchi Y, et al: MicroRNA profiling of human intrahepatic cholangiocarcinoma cell lines reveals biliary epithelial cell-specific microRNAs. J Nippon Med Sch 76: 188-197, 2009.

6. Isomoto H: Epigenetic alterations associated with cholangiocarcinoma (Review). Oncol Rep 22: 227-232, 2009.

7. Chen L, Yan HX, Yang W, et al: The role of microRNA expression pattern in human intrahepatic cholangiocarcinoma. J Hepatol 50: 358-369, 2009.

8. Zhong XY, Yu JH, Zhang WG, et al: MicroRNA-421 functions as an oncogenic miRNA in biliary tract cancer through downregulating farnesoid X receptor expression. Gene 493: 44-51, 2012.

9. Zhang J, Han C and Wu T: MicroRNA-26a promotes cholangiocarcinoma growth by activating $\beta$-catenin. Gastroenterology 143 : 246-256.e248, 2012.

10. Yamanaka S, Campbell NR, An F, et al: Coordinated effects of microRNA-494 induce $\mathrm{G}(2) / \mathrm{M}$ arrest in human cholangiocarcinoma. Cell Cycle 11: 2729-2738, 2012.

11. Razumilava N, Bronk SF, Smoot RL, et al: miR-25 targets TNF-related apoptosis inducing ligand (TRAIL) death receptor-4 and promotes apoptosis resistance in cholangiocarcinoma. Hepatology 55: 465-475, 2012.

12. Oishi N, Kumar MR, Roessler S, et al: Transcriptomic profiling reveals hepatic stem-like gene signatures and interplay of miR-200c and epithelial-mesenchymal transition in intrahepatic cholangiocarcinoma. Hepatology 56: 1792-1803, 2012.

13. Liu CZ, Liu W, Zheng Y, et al: PTEN and PDCD4 are bona fide targets of microRNA-21 in human cholangiocarcinoma. Chin Med Sci J 27: 65-72, 2012.

14. He Q, Cai L, Shuai L, et al: Ars2 is overexpressed in human cholangiocarcinomas and its depletion increases PTEN and PDCD4 by decreasing MicroRNA-21. Mol Carcinog: Dec 28, 2011 (Epub ahead of print). 
15. Selaru FM, Olaru AV, Kan T, et al: MicroRNA-21 is overexpressed in human cholangiocarcinoma and regulates programmed cell death 4 and tissue inhibitor of metalloproteinase 3. Hepatology 49: 1595-1601, 2009.

16. Li B, Han Q, Zhu Y, Yu Y, Wang J and Jiang X: Down-regulation of miR-214 contributes to intrahepatic cholangiocarcinoma metastasis by targeting Twist. FEBS J 279: 2393-2398, 2012.

17. Chen YJ, Luo J, Yang GY, Yang K, Wen SQ and Zou SQ Mutual regulation between microRNA-373 and methyl-CpGbinding domain protein 2 in hilar cholangiocarcinoma. World J Gastroenterol 18: 3849-3861,2012.

18. Chen Y, Luo J, Tian R, Sun H and Zou S: miR-373 negatively regulates methyl-CpG-binding domain protein 2 (MBD2) in hilar cholangiocarcinoma. Dig Dis Sci 56: 1693-1701, 2011.

19. Chen Y, Gao W, Luo J, Tian R, Sun H and Zou S: Methyl-CpG binding protein MBD2 is implicated in methylation-mediated suppression of miR-373 in hilar cholangiocarcinoma. Oncol Rep 25: 443-451, 2011.

20. Olaru AV, Ghiaur G, Yamanaka S, et al: MicroRNA down-regulated in human cholangiocarcinoma control cell cycle through multiple targets involved in the G1/S checkpoint. Hepatology 54 2089-2098, 2011.

21. Meng F, Wehbe-Janek H, Henson R, Smith H and Patel T: Epigenetic regulation of microRNA-370 by interleukin- 6 in malignant human cholangiocytes. Oncogene 27: 378-386, 2008.

22. Lee ST, Chu K, Im WS, et al: Altered microRNA regulation in Huntington's disease models. Exp Neurol 227: 172-179, 2011.

23. Jiang L, Liu X, Kolokythas A, et al: Downregulation of the Rho GTPase signaling pathway is involved in the microRNA-138-mediated inhibition of cell migration and invasion in tongue squamous cell carcinoma. Int J Cancer 127: 505-512, 2010

24. Liu X, Chen Z, Yu J, Xia J and Zhou X: MicroRNA profiling and head and neck cancer. Comp Funct Genomics 2009: 837514, 2009.

25. Mitomo S, Maesawa C, Ogasawara S, et al: Downregulation of miR-138 is associated with overexpression of human telomerase reverse transcriptase protein in human anaplastic thyroid carcinoma cell lines. Cancer Sci 99: 280-286, 2008.

26. Poliseno L, Haimovic A, Segura MF, et al: Histology-specific microRNA alterations in melanoma. J Invest Dermatol 132: 1860-1868, 2012

27. Jiang L, Dai Y, Liu X, et al: Identification and experimental validation of $G$ protein alpha inhibiting activity polypeptide 2 (GNAI2) as a microRNA-138 target in tongue squamous cell carcinoma. Hum Genet 129: 189-197, 2011.

28. Wang W, Zhao LJ, Tan YX, Ren H and Qi ZT: MiR-138 induces cell cycle arrest by targeting cyclin D3 in hepatocellular carcinoma. Carcinogenesis 33: 1113-1120, 2012.

29. Liu X, Lv XB, Wang XP, et al: MiR-138 suppressed nasopharyngeal carcinoma growth and tumorigenesis by targeting the CCND1 oncogene. Cell Cycle 11: 2495-2506, 2012.
30. Clark EA, Golub TR, Lander ES and Hynes RO: Genomic analysis of metastasis reveals an essential role for RhoC. Nature 406: 532-535, 2000.

31. Iiizumi M, Bandyopadhyay S, Pai SK, et al: RhoC promotes metastasis via activation of the Pyk 2 pathway in prostate cancer. Cancer Res 68: 7613-7620, 2008.

32. Shi Z, Chen ML, He QL and Zeng JH: Antisense RhoC gene suppresses proliferation and invasion capacity of human QBC939 cholangiocarcinoma cells. Hepatobiliary Pancreat Dis Int 6: 516-520, 2007.

33. Arocho A, Chen B, Ladanyi M and Pan Q: Validation of the 2-DeltaDeltaCt calculation as an alternate method of data analysis for quantitative PCR of BCR-ABL P210 transcripts. Diagn Mol Pathol 15: 56-61, 2006.

34. Saadoun S, Papadopoulos MC, Hara-Chikuma $M$ and Verkman AS: Impairment of angiogenesis and cell migration by targeted aquaporin-1 gene disruption. Nature 434: 786-792, 2005.

35. Aspenstrom P, Fransson A and Saras J: Rho GTPases have diverse effects on the organization of the actin filament system. Biochem J 377: 327-337, 2004.

36. Ravenhall C, Guida E, Harris T, Koutsoubos V and Stewart A: The importance of ERK activity in the regulation of cyclin D1 levels and DNA synthesis in human cultured airway smooth muscle. Br J Pharmacol 131: 17-28, 2000.

37. Corona G, Deiana M, Incani A, Vauzour D, Dessi MA and Spencer JP: Hydroxytyrosol inhibits the proliferation of human colon adenocarcinoma cells through inhibition of ERK1/2 and cyclin D1. Mol Nutr Food Res 53: 897-903, 2009.

38. Ikoma T, Takahashi T, Nagano S, et al: A definitive role of RhoC in metastasis of orthotopic lung cancer in mice. Clin Cancer Res 10: 1192-1200, 2004.

39. Xue F, Takahara T, Yata Y, et al: Blockade of Rho/Rho-associated coiled coil-forming kinase signaling can prevent progression of hepatocellular carcinoma in matrix metalloproteinase-dependent manner. Hepatol Res 38: 810-817, 2008.

40. van Golen KL, Wu ZF, Qiao XT, Bao LW and Merajver SD: RhoC GTPase, a novel transforming oncogene for human mammary epithelial cells that partially recapitulates the inflammatory breast cancer phenotype. Cancer Res 60: 5832-5838, 2000.

41. Blain EJ: Mechanical regulation of matrix metalloproteinases. Front Biosci 12: 507-527, 2007.

42. Ehrenfeld P, Conejeros I, Pavicic MF, et al: Activation of kinin B1 receptor increases the release of metalloproteases-2 and -9 from both estrogen-sensitive and -insensitive breast cancer cells. Cancer Lett 301: 106-118, 2011.

43. Ming J, Liu N, Gu Y, Qiu X and Wang EH: PRL-3 facilitates angiogenesis and metastasis by increasing ERK phosphorylation and up-regulating the levels and activities of Rho-A/C in lung cancer. Pathology 41: 118-126, 2009. 